\title{
LA ADMINISTRACIÓN ESTRATÉGICA Y LA PRODUCTIVIDAD EN LAS AGENCIAS DE VIAJES Y TURISMO
}

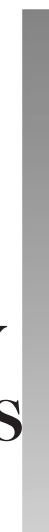

Dr. Jorge V. Mayurí Barrón* jvmayuri@gmail.com

\begin{abstract}
RESUMEN
El uso de la Administración Estratégica o Planeación Estratégica como herramienta a través de sus matrices, permite que este estudio de aplicación para las agencias de viajes y turismo mejoren su productividad debido a la alta competencia en el mercado y, así mismo, por la reducción de comisiones por parte de las aerolíneas nacionales e internacionales y algunos prestatarios de la industria turística, obligando a mejorar la calidad de gestión por parte de las agencias de viajes y turismo.

Las estadísticas obtenidas a través del Ministerio de Comercio Exterior y Turismo (Mincetur) determinaron un total de 2,383 agencias existentes en la ciudad de Lima, de las cuales se ha tomado como referencia 10 de las mejores agencias que operan turismo receptivo, egresivo e interno, y que al mismo tiempo son agentes de pasaje IATA y en algunos casos operan como tour operadoras.

Se demuestra en los resultados que 7 de las 10 agencias manejan Planeamiento Estratégico y que por esta razón llegaron a incrementar su productividad
\end{abstract}

Palabras claves: planeación, productividad, agencia de viajes, turismo.

\begin{abstract}
The use of Strategic Management or Strategic Planning as a tool through its matrix allows this study to be applied to Travel Agencies to improve their productivity due to high competition in the market and also for the reduction of commissions from national and international airlines, and some tourism industry borrowers, forcing to improve the quality of management by travel agencies.

Statistics obtained through MINCETUR (Ministry of Foreign Trade and Tourism) identified a total of 2.383 existing travel agencies in the city of Lima taking as reference 10 of the best travel agencies operating inbound tourism, domestic and international at the same time, the travel agencies are IATA Passenger Agents and sometimes operating as tour operators.

Results demonstrates that 7 of 10 agencies handle strategic planning, and for this reason they came to increase productivity
\end{abstract}

Keywords: Planning, Productivity, travel agencies, tourism.

\footnotetext{
* Doctor en Educación (UNMSM), Magister en Administración, con Mención en Gestión Empresarial (UNMSM); Licenciado en Administración de Empresas (Universidad Ricardo Palma) Post Grado en Derecho y Espacial (UNMSM), Profesor Asociado de la Facultad de Ciencias Administrativas de la UNMSM, Profesor de Post Grado en: USMP, UNFV, UNSCH, UNCP, Capacitador Empresarial.
} 


\section{INTRODUCCIÓN}

Las agencias de viaje y turismo en nuestro país pertenecen a un sector de enorme importancia para la economía de la nación. De ellas, se extrae el $10 \%$ del PBI, siendo una fuente generadora de recursos innegable.

La apertura y creación de nuevos destinos turísticos en nuestro país, y los tratados de libre comercio y la globalización del mercado, hacen que el Perú, en muchas de sus regiones, sea un destino turístico único, haciendo que muchas micro y pequeñas empresas se desarrollen en este rubro.

Dada la alta competencia, es necesario que la gran mayoría de ellas empiecen a utilizar herramientas no solo de sobrevivencia a corto plazo, sino de competencia para existir y ofrecer mejores productos, tal es el caso de La Planeación estratégica.

\section{MARCO TEÓRICO}

Toda empresa diseña planes estratégicos para el logro de sus objetivos y metas planteadas, esto planes pueden ser a corto, mediano y largo plazo, según la amplitud y magnitud de la empresa. Es decir, su tamaño, ya que esto implica que cantidad de planes y actividades debe ejecutar cada unidad operativa, ya sea de niveles superiores o niveles inferiores.

Ha de destacarse que el presupuesto refleja el resultado obtenido de la aplicación de los planes estratégicos. Es de considerarse que es fundamental conocer y ejecutar correctamente los objetivos para poder lograr las metas trazadas por las empresas.

También es importante señalar que la empresa debe precisar con exactitud y cuidado la misión que se va regir la empresa, la misión es fundamental, ya que esta representa las funciones operativas que va a ejecutar en el mercado y va a suministrar a los consumidores.

La planificación estratégica constituye un sistema gerencial que desplaza el énfasis en el "qué lograr" (objetivos) al "qué hacer" (estrategias). Con la planificación estratégica se busca concentrarse en solo aquellos objetivos factibles de lograr y en qué negocio o área competir, en correspondencia con las oportunidades y amenazas que ofrece el entorno.

Hace falta impulsar el desarrollo cultural, esto significa que todas las personas relacionadas con la organización se desarrollen en su saber, en sus expectativas, en sus necesidades, y en sus formas de relacionarse y de enfrentar al mundo presente y futuro, esencialmente dinámico.

Se consideran cuatro puntos de vista en la planeación estratégica:

\section{El porvenir de las decisiones actuales}

Primero, la planeación trata con el porvenir de las decisiones actuales. Esto significa que la planeación estratégica observa la cadena de consecuencias de causas y efectos durante un tiempo, relacionada con una decisión real o intencionada que tomará el director. La esencia de la planeación estratégica consiste en la identificación sistemática de las oportunidades y peligros que surgen en el futuro, los cuales combinados con otros datos importantes proporcionan la base para qué una empresa tome mejores decisiones en el presente para explotar las oportunidades y evitar los peligros. Planear significa diseñar un futuro deseado e identificar las formas para lograrlo.

\section{Proceso}

Segundo, la planeación estratégica es un proceso que se inicia con el establecimiento de metas organizacionales, define estrategias y políticas para lograr estas metas, y desarrolla planes detallados para asegurar la implantación de las estrategias y así obtener los fines buscados. También es un proceso para decidir de antemano qué tipo de esfuerzos de planeación debe hacerse, cuándo y cómo debe realizarse, quién lo llevará a cabo, y qué se hará con los resultados. La planeación estratégica es sistemática en el sentido de que es organizada y conducida con base en una realidad entendida.

Para la mayoría de las empresas, la planeación estratégica representa una serie de planes producidos después de un tiempo específico, durante el cual se elaboraron los planes. También debería entenderse como un proceso continuo, especialmente en cuanto a la formulación de estrategias, ya que los cambios en el ambiente del negocio son continuos. La idea no es que los planes deberían cambiarse a diario, sino que la planeación debe efectuarse en forma continua y ser apoyada por acciones apropiadas cuando sea necesario.

\section{Filosofía}

Tercero, la planeación estratégica es una actitud, una forma de vida; requiere de dedicación para actuar con base en la observación del futu- 
ro, y una determinación para planear contante y sistemáticamente como una parte integral de la dirección. Además, representa un proceso mental, un ejercicio intelectual, más que una serie de procesos, procedimientos, estructuras o técnicas prescritos.

\section{Estructura}

Cuarto, un sistema de planeación estratégica formal une tres tipos de planes fundamentales, que son: planes estratégicos, programas a mediano plazo, presupuestos a corto plazo y planes operativos. La planeación estratégica es el esfuerzo sistemático y más o menos formal de una compañía para establecer sus propósito, objetivos, políticas y estrategias básicas, para desarrollar planes detallados con el fin de poner en práctica las políticas y estrategias y así lograr los objetivos y propósitos básicos de la compañía.

\section{Lo que no es la planeación estratégica}

La planeación estratégica no trata de tomar decisiones futuras, ya que estas solo pueden tomarse en el momento. La planeación del futuro exige que se haga la elección entre posibles sucesos futuros, pero las decisiones en sí, las cuales se toman con base en estos sucesos, solo pueden hacerse en el momento.

La planeación estratégica no pronostica las ventas de un producto para después determinar qué medidas tomar con el fin de asegurar la realización de tal pronóstico en relación con factores tales como: compras de material, instalaciones, mano de obra, etc.

La planeación estratégica no representa una programación del futuro, ni tampoco el desarrollo de una serie de planes que sirvan de molde para usarse diariamente sin cambiarlos en el futuro lejano. Una gran parte de empresas revisa sus planes estratégicos en forma periódica, en general una vez al año. La planeación estratégica debe ser flexible para poder aprovechar el conocimiento acerca del medio ambiente.

La planeación estratégica no representa esfuerzo para sustituir la intuición y criterio de los directores.

La planeación estratégica no es nada más un conjunto de planes funcionales o una extrapolación de los presupuestos actuales; es un enfoque de sistemas para guiar una empresa durante un tiempo a través de su medio ambiente, para lograr las metas dictadas.

\section{Modelos conceptuales de la planeación estra-} tégica

Un modelo conceptual es aquel que presenta una idea de lo que algo debería ser en general, o una imagen de algo formado mediante la generalización de particularidades. A cambio, un modelo operativo es el que se usa en realidad en las empresas.

Las principales características de los modelos conceptuales de la planeación estratégica son:

\section{Premisas de planeación}

Premisas significa literalmente lo que va antes, lo que se establece con anterioridad, o lo que se declara como introductorio, postulado o implicado, las premisas están divididas en dos tipos: plan para planear, y la información sustancial, necesaria para el desarrollo e implantación de los planes.

Antes de llevar a cabo un programa estratégico de planeación es importante que las personas involucradas en él tengan un amplio conocimiento de lo que tiene en mente el alto directivo y cómo operará el sistema. Esta guía está incorporada en un plan para planear, el cual puede ser oral, aunque usualmente es escrito, para su distribución general.

La información acumulada en estas áreas algunas veces es llamada "análisis de situación", pero también se usan otros términos para denominar esta parte de la planeación.

Ninguna organización, no importa cuan grande o lucrativa sea, puede examinar en forma minuciosa todos los elementos que posiblemente están incluidos en el análisis de la situación. Es por este motivo que cada organización debe identificar aquellos elementos - pasados, presentes y futuros - que son de gran importancia para su crecimiento, prosperidad y bienestar, y debe concentrar su pensamiento y sus esfuerzos para entenderlos. Otros elementos se pueden considerar en esta parte del proceso de la planeación aunque pueden ser estimados sin ser investigados o sacados de documentos publicados al respecto.

\section{Formulación de planes}

En cuanto a conceptos y con base en las premisas antes mencionadas, el siguiente paso en el proceso de planeación estratégica es formular estrategias maestras y de programas. Las estrategias maestras se definen como misiones, propósitos, objetivos y políticas básicas; mientras que 
las estrategias de programa se relacionan con la adquisición, uso y disposición de los recursos para proyectos específicos, tales como la construcción de una nueva planta en el extranjero.

A diferencia de la programación a mediano plazo no existe un enfoque modelo para planear en esta área. Lo que se hace depende de los deseos de los directores en un momento dado, los cuales, a su vez, son estimulados por las condiciones a las que se enfrenta la empresa en un momento preciso.

La programación a mediano plazo es el proceso mediante el cual se prepara y se interrelacionan planes específicos funcionales para mostrar los detalles de cómo se debe llevar a cabo le estrategia para lograr objetivos, misiones y propósitos de la compañía a largo plazo. El periodo típico de planeación es de cinco años, pero existe una tendencia en las compañías más avanzadas en cuanto a tecnología, de planear por adelantado de siete a diez años. Las empresas que se enfrentan a ambientes especialmente problemáticos algunas veces reducen la perspectiva de planeación a cuatro o tres años.

El siguiente paso es desarrollar los planes a corto plazo con base en los planes a mediano plazo. En algunas organizaciones los números obtenidos durante el primer año de los planes a mediano plazo son los mismos que aquellos logrados con los planes operativos anuales a corto plazo, aunque en otras empresas no existe la misma similitud. Los planes operativos serán mucho más detallados que los planes de programación a mediano plazo.

\section{Implementación y revisión}

Una vez que los planes operativos son elaborados deben ser implantados. El proceso de implantación cubre toda la gama de actividades directivas, incluyendo la motivación, compensación, evaluación directiva y procesos de control.

Los planes deben ser revisados y evaluados. No existe mejor manera para producir planes por parte de los subordinados que cuando los altos directivos muestran un interés profundo en estos $\mathrm{y}$ en los resultados que pueden producir.

Cuando fue desarrollada por primera vez la planeación formal en la década de los cincuenta, las compañías tendían a hacer planes por escrito y no revisarlos hasta que obviamente eran obsoletos. En la actualidad, la gran mayoría de las empresas pasa por un ciclo anual de planeación, durante el cual se revisan los planes. Este proceso debería contribuir significativamente al mejoramiento de la planeación del siguiente ciclo.

\section{Flujos de información y normas de evaluación y decisión}

Los "flujos de información" simplemente deben transmitir el punto de que la información "fluye" por todo el proceso de planeación. Este "flujo" difiere grandemente, dependiendo de la parte del proceso a la que sirve y del tema de la información.

En todo proceso de planeación es necesario aplicar las normas de decisión y evaluación. Por otra parte, con el desarrollo de los planes operativos actuales, las normas de decisión se convierten en más cuantitativas, o sea, en fórmulas de sustitución de inventarios o de rendimientos sobre inversión.

La planificación estratégica es una herramienta por excelencia de la gerencia estratégica. Consiste en la búsqueda de una o más ventajas competitivas de la organización y la formulación y puesta en marcha de estrategias, permitiendo crear o preservar sus ventajas, todo esto en función de la misión y de sus objetivos, del medio ambiente y sus presiones y de los recursos disponibles.

Sallenave (1991) afirma que "La planificación estratégica es el proceso por el cual los dirigentes ordenan sus objetivos y sus acciones en el tiempo. No es un dominio de la alta gerencia, sino un proceso de comunicación y de determinación de decisiones en el cual intervienen todos los niveles estratégicos de la empresa".

La planificación estratégica tiene por finalidad producir cambios profundos en los mercados de la organización y en la cultura interna.

La expresión planificación estratégica es un plan estratégico corporativo, el cual se caracteriza fundamentalmente por coadyuvar a la racionalización de la toma de decisiones, se basa en la eficiencia institucional e integra la visión de largo plazo (filosofía de gestión), mediano plazo (planes estratégicos funcionales) y corto plazo (planes operativos).

Es el conjunto de actividades formales encaminadas a producir una formulación estratégica. Estas actividades son de muy variado tipo y van desde una reunión anual de directivos para discutir las metas para el ejercicio entrante, hasta 
la obligatoria recopilación y envío de datos presupuestarios por parte de todas las unidades de la empresa a la unidad superior. Son aquellos mecanismos formales, es decir, de obligado cumplimiento a plazo fijo, que "fuerzan" el desarrollo de un plan estratégico para la empresa.

Aporta una metodología al proceso de diseño estratégico, guían a la dirección en la tarea de diseñar la estrategia.

La planificación estratégica no es solo una herramienta clave para el directivo implica, necesariamente, un proceso interactivo de arriba abajo y de abajo arriba en la organización; la dirección general marca metas generales para la empresa (apoyada en la información de mercados recibida, con seguridad, de las unidades inferiores) y establece prioridades; las unidades inferiores determinan planes y presupuestos para el período siguiente; esos presupuestos son consolidados y corregidos por las unidades superiores, que vuelven a enviarlos hacia abajo, donde son nuevamente retocados, etc. Como consecuencia, el establecimiento de un sistema formal de planificación estratégica hace descender la preocupación estratégica a todos los niveles de la organización.

\section{Planeación estratégica}

La empresa selecciona, entre varios caminos alternativos, el que considera más adecuado para alcanzar los objetivos propuestos. Generalmente, es una planeación global a largo plazo.

La planeación estratégica exige cuatro fases bien definidas: formulación de objetivos organizacionales; análisis de las fortalezas y limitaciones de la empresa; análisis del entorno; formulación de alternativas estratégicas.

\section{Planificación estratégica: utilización del proceso.}

La planeación estratégica es el proceso administrativo de desarrollar y mantener una relación viable entre los objetivos recursos de la organización y las cambiantes oportunidades del mercado. El objetivo de la planeación estratégica es modelar y remodelar los negocios y productos de la empresa, de manera que se combinen para producir un desarrollo y utilidades satisfactorios.

La planeación estratégica y su conjunto de conceptos y herramientas no surgieron sino hasta principios de la década de los años sesenta. Anteriormente la administración se las arreglaba bastante bien con la planeación de operaciones pues, con el crecimiento continuo de la demanda total era difícil estropear los negocios, aun en el caso de administraciones deficientes. Pero entonces estallaron los turbulentos años 70. Y hubo una sucesión de crisis: Los precios del petróleo se dispararon como consecuencia de la guerra en el Medio Oriente. Sobrevino una escasez de materiales y energía, acompañada de una inflación de dos dígitos y luego el estancamiento económico y el aumento del desempleo. Mercaderías de bajo costo y alta calidad procedente de Japón y otros lugares empezaron a invadir principalmente a Estados Unidos, apoderándose de las participaciones de industrias muy fuertes, como las del acero, automóviles, motocicletas, relojes y cámaras fotográficas. Todavía, posteriormente, algunas empresas tuvieron que vérselas con una creciente ola de irregularidades en industrias claves como las de telecomunicaciones, transporte, energía, servicios, de salud, leyes, y contabilidad. Las empresas que habían funcionado con las antiguas reglas, se enfrentaban ahora a una intensa competencia doméstica y externa que desafiaba sus venerables prácticas de negocios.

Esta sucesión de sacudidas hizo necesario un nuevo proceso de planeación de la administración para mantener saludables las empresas, a pesar de los trastornos ocurridos en cualquiera de sus negocios o líneas de productos.

La planificación estratégica proporciona la dirección que guiará la misión, los objetivos y las estrategias de la empresa, pues facilita el desarrollo de planes para cada una de sus áreas funcionales. Un plan estratégico completo guía cada una de las áreas en la dirección que la organización desea seguir y les permite desarrollar objetivos, estrategias y programas adecuados a las metas. La relación entre la planificación estratégica y la de operaciones es parte importante de las tareas de la gerencia.

\section{El proceso de la planeación estratégica}

(Plan estratégico. Aplicación en un concepto determinado).

\section{Ejemplo:}

Detrás de cada acción reflexionada existe una voluntad. ¿De dónde proviene la chispa inicial de voluntad que dará lugar al desarrollo de un plan para llegar finalmente a la acción empresarial?: De una visión. 


\section{Etapa \# 1 Visión Misión}

La palabra visión evoca imágenes sobrenaturales, apariciones, profecías y revelaciones, y a los visionarios los sitúan en el panorama social al lado de los locos. Sin embargo, toda empresa, todo plan, empieza con una visión.

En los años setenta, la empresa Xerox invitó a un joven ingeniero llamado Steve Jobs a que asistiera con otras personas a la demostración de una innovación todavía sin aplicación práctica: un operador desplazaba una pequeña máquina en una mesita, y se podían seguir sus movimientos en la pantalla. Todos pensaron que la máquina era ingeniosa. Solo Steve Jobs tuvo la visión. Se dio cuenta de inmediato de que la invención del "ratón" (Computer mouse) podía revolucionar la forma en que se utilizaban los computadores. Es esta visión nació Macintosh, y creció la compañía Apple Computers.

La visión es más bien una manera distinta de ver las cosas, es la percepción simultánea de un problema y de una solución técnica novedosa; al fin y al cabo es una apuesta sobre la aceptación de una idea por el público. La visión adscribe una misión a la empresa: hacer que la visión se convierte en realidad.

Cuando una empresa formula su misión, no trata de hacer la publicidad de sus productos; tampoco les dirige un discurso filosófico a los accionistas sino que aclara sus propósitos, identifica su razón de ser y define su negocio en todas sus dimensiones. La responsabilidad de la empresa establece en función de la utilidad social, lo cual implica un juicio de valor sobre la contribución eventual de la empresa a un conjunto de objetivos sociales o político - económicos, mientras que la misión de la empresa no es más que una delimitación del campo de acción que los dirigentes le fijan a priori a la empresa.

La definición de una misión delimita, el campo de actividades posibles, con el fin de concentrar los recursos de la empresa en un campo general o dirigirlos hacia un objetivo permanente.

Para el gerente, la misión es lo que la cancha es para el futbolista. Enmarca su acción y sus movimientos (estrategias). El juego no puede desarrollarse fuera de la cancha. Por lo general, fracasan las compañías que comenten el error de salirse de la cancha y lanzarse a negocios que no corresponden a su misión.

\section{Proceso de la planeación estratégica}

Desarrollar misiones y propósitos comerciales básicos

\section{El conjunto de las metas comerciales}

Los propósitos y las misiones comerciales básicas son la base para el conjunto de metas en un negocio. No existe uniformidad en la estructura del mismo en el mundo de los negocios, sino lo que se incluye o excluye, la manera en que se ordenan y se relacionan las metas varía de una compañía a otra, y de un momento a otro dentro de la misma empresa.

\section{Tipos de metas}

Los planes estratégicos, son diseñados por los altos ejecutivos y los administradores de mandos medios para lograr las metas generales de la organización

Los planes operacionales. Son aquellos que indican cómo serán implantados los planes estratégicos mediante las actividades diarias.

\section{Doctrinas o filosofías de una empresa}

En años recientes ha habido un rápido aumento en las premisas de propósitos y misiones publicados de la compañía. Estos documentos son denominados: doctrinas, políticas, estrategias, propósitos de la empresa, filosofías y los observadores más críticos los llaman relaciones públicas engañosas. Estas premisas generalmente expresan los propósitos básicos de una compañía y las creencias de los ejecutivos en jefe.

Por esto, un propósito básico para cualquier negocio es buscar la utilidad mediante el uso efectivo y eficaz de recursos.

La meta de utilidad puede tomar varias formas:

"Nuestro propósito es crear suficientes utilidades para mantener el bienestar y crecimiento de la compañía”.

"La meta es optimizar las utilidades".

"Un propósito básico (de nuestra compañía) es proporcionar un alto nivel de crecimiento de las ganancias por acciones".

"Nuestro objetivo básico es obtener y pagar dividendos a nuestros accionistas y cuidar su inversión". 


\section{Misiones básicas}

Las premisas de las misiones identifican el diseño, meta o empuje implícitos de una compañía. Pueden ser expresadas a diferentes niveles de abstracción.

\section{Importancia de las premisas de misiones}

Las premisas además de proporcionar guías generales para la planeación estratégica, son importantes para la creación de estrategias programadas y la naturaleza de un negocio; determinan el área de competencia donde opera el mismo; establecen la forma en que serán distribuidos los recursos de acuerdo las diferentes solicitudes; determinan el tamaño de la compañía; facilitan la identificación de oportunidades y peligros que deben ser tratados en el proceso de planeación; ofrecen nuevas oportunidades al igual que peligros y evitan que la gente "pierda su tiempo" trabajando con estrategias y planes que puedan ser considerados inapropiados por la alta dirección.

\section{¿Cuál es la misión apropiada?}

Solo se puede determinar si una misión es "correcta» o no hasta después de haber tomado la decisión. La determinación de una misión está basada en el juicio. Como dice Vickers: «El valor de los juicios del hombres y la sociedad no prueban ser correctos o incorrectos: sino que solo pueden ser aprobados como correctos o condenados como equivocados mediante otro juicio de valor».

\section{¿Cómo se formulan las misiones?}

No existe un enfoque estándar para la creación de misiones; las premisas de misiones dependen fuertemente de los valores del EJ y no pueden cambiarse fácilmente sin la intervención directa del mismo.

Cambiar significativamente una misión o un propósito básico requerirá de otros cambios importantes en las operaciones de la organización, en las relaciones mutuas con la gente, y en la manera en que ésta utiliza sus habilidades, etc. Como consecuencia, los cambios precipitados, a menos de que se hagan en respuesta a una crisis, tienden a tener menos éxito que aquellos que son analizados gradualmente a través de pláticas extensas.

Secuencia de pasos: primero, reconocían una necesidad para un cambio; segundo, buscaban que la organización conociera esta necesidad al comi- sionar grupos de trabajo, miembros del personal o asesores para examinar problemas, opciones, contingencias y oportunidades producidas por la necesidad percibida; tercero, trataron de ampliar el apoyo mediante pláticas no estructuradas, prueba de posiciones, definición de diferencias de opinión, alentando los conceptos favorecidos por el EJ, desalentando ideas no favorecidas por éste y la alta dirección, etc.; cuarto, creaban obligaciones al crear las habilidades o tecnologías dentro de la organización, probando opciones y utilizando oportunidades para tomar decisiones que aumentan el apoyo; quinto, establecían un claro enfoque, ya sea al crear un comité ad hoc para formular una posición, o al expresar fines específicos que la alta dirección deseaba; sexto, obtuvieron un buen cumplimiento al asignar a alguien el logro del objetivo, y la responsabilidad de su logro. Este último paso puede ampliarse, por ejemplo, al incluir obligaciones específicas en presupuestos y al hacer planes operativos a corto plazo.

\section{¿Deben hacerse por escrito las misiones y los propósitos?}

Si existe un acuerdo general entre los directores acerca de las premisas de misión y propósito, tenerlos por escrito puede ayudar a reforzar su cumplimiento durante el paso del tiempo. Por otro lado, cuando las palabras están escritas pueden generar rápidamente malentendidos y disputas.

\section{Tipos conceptuales y operativos de objetivos}

En teoría, los objetivos deberían establecerse para cada elemento de una empresa los cuales según la alta dirección deben ser sujetos a planes. No existe una clasificación estándar de objetivos o de la cantidad de los mismos que debería tener una empresa.

En la práctica, la mayoría de las compañías tienen relativamente pocos objetivos de planeación a largo plazo. Generalmente establecen los mismos para ventas, utilidades se expresan por lo general en cifras absolutas o porcentaje, o ambos.

Otras áreas para las cuales se establecen objetivos incluyen: desarrollo del producto, productividad, diversificación, contratación de minorías, sustitución de instalaciones, intensidad de trabajo del producto, clasificación de la industria, desarrollo directivo, condiciones laborales, niveles de empleo y responsabilidades sociales. 
Peter Drucker dijo «que los objetivos son necesarios para cada área de negocio cuyo desempeño y resultado directamente afectan la supervivencia y prosperidad de la empresa. Identificó las siguientes áreas como aquellas que necesitan un objetivo: posición del mercado, innovación, productividad, recursos financieros y físicos, utilidad, desempeño y desarrollo directivos, desempeño y actitud de los trabajadores, y responsabilidad pública».

\section{La relación entre objetivos}

Los objetivos y los subobjetivos deberían estar muy relacionados, ya que en las operaciones actuales de una empresa existe una estrecha relación entre los objetivos económicos dominantes.

\section{Procesos para establecer objetivos}

Existen cuatros enfoques fundamentalmente diferentes para plantear el enfoque ascendiente, el descendiente, una combinación de estos dos, y el enfoque en equipo. En compañías muy pequeñas, el proceso para establecer un objetivo generalmente es descendiente, pero en la mayoría de las empresas grandes se emplea una combinación entre el ascendiente y descendiente o el enfoque en equipo En compañías pequeñas y en unidades comerciales estratégicas de grandes corporaciones este proceso se realiza frecuentemente mediante un esfuerzo en equipo.

En empresas más grandes y diversificadas, un modelo únicamente descendiente para establecer un objetivo sería inadecuadamente por dos razones: primero, la alta dirección no tiene los conocimientos suficientes acerca de todos los negocios con los que trata la compañía para establecer metas reales; segundo, el típico gerente de división resentiría si se le diera un objetivo sin que él tuviera la oportunidad de discutir su factibilidad (una razón práctica que explica su actitud es que el establecer objetivos es un proceso muy complejo que involucra todo tipo de intercambios simbólicos). Por el otro lado, existen pocos directores, si del todo, dispuestos a aceptar que se establezcan objetivos divisionales sin su revisión y su aprobación. Por estas razones, el procedimiento para establecer el objetivo en empresa más grandes generalmente involucra la cooperación entre la alta dirección y los gerentes de divisiones.

\section{La planificación a largo plazo}

La planificación a largo plazo, obliga a pensar en el futuro, hay que tener una idea de qué es lo que estará haciendo entonces la empresa, a dónde se habrá dirigido el sector, y cuáles serán las posiciones relativas de los competidores y realizar un serio análisis estratégico.

La fuerte turbulencia económica de los últimos años ha llevado a algunos directivos a abandonar la planificación a largo plazo, pensando que es sensillamanete imposible. plazo

Desarrollar objetivos de planeación a largo

El significado de objetivos de planeación a largo plazo

Un objetivo, se refiere a un resultado que se desea o necesita lograr dentro de un periodo de tiempo específico. Es un valor aspirado por un individuo o un grupo dentro de una organización; una clase específica de un propósito fundamental y define en forma más concreta a éste o a una parte del mismo; es un estado futuro deseado de un negocio o de uno de sus elementos. A pesar de que el objetivo debe lograrse en el futuro, se determina un lapso específico para su realización.

\section{Criterios para los objetivos:}

\section{Conveniente}

Un requisito obvio para un objetivo es que su logro debe apoyar los propósitos y misiones básicos de una empresa. Además, debe guiar a la misma en la dirección identificada por los propósitos y misiones básicos. Un objetivo que no hace ninguna contribución al propósito no es productivo, y uno que se opone al mismo es peligroso. Una contribución positiva a un propósito debe ser planeada si el objetivo debe tener un efecto más que casual en esta área.

\section{Mensurable a través del tiempo}

Hasta donde sea posible, los objetivos deberán establecer, en términos concretos, lo que se espera que ocurra y cuándo. La planeación se facilita bastante cuando los objetivos están fijados no como generalidades inexactas sino en términos concretos. Los objetivos pueden cuantificarse en términos, tales como calidad, cantidad, tiempo, costo, índice, porcentaje, tasa, o en pasos específicos a seguirse.

\section{Factible}

Los directivos no deberían establecer objetivos poco prácticos o irreales, sino que éstos deberían ser posibles de lograr; sin embargo, no es fácil 
fijar objetivos prácticos, ya que numerosas consideraciones están involucradas en el proceso de su determinación. El objetivo debe establecerse en vista de lo que los directivos consideran que pasará en la rama industrial: las posibles acciones de los competidores y las proyecciones en los aspectos económicos, sociales, políticos y técnicos del medio ambiente. Además, deben tomarse en cuenta los factores internos, tales como capacidades directivas, fuerzas que motivan o desaniman a empleados, capital disponible y habilidades de innovación técnica. Un objetivo factible refleja de manera realista tales fuerzas, las cuales con la realización apropiada de los planes pueden esperarse que ocurren dentro del lapso especificado.

\section{Aceptable}

Los objetivos pueden lograse más fácilmente si son aceptables para las personas dentro de una organización. Un objetivo que no se adapta al sistema de valores de un director importante, no será aspirado asiduamente. Trátese de hacer responsable a un directivo pro digamos desarrollar un nuevo producto que al director no le agrada. El objetivo también debería ser aceptable en cuanto que la empresa ésta de acuerdo en incurrir en costos necesarios para su logro. Costos en este sentido no solo significan recursos financieros sino que también el tiempo de los directivos y del personal, capacidad de la planta, participación del mercado y utilidades utilizados durante un lapso específico.

\section{Flexible}

Debería ser posible modificar el objetivo cuando surgen contingencias inesperadas, aunque no debería ser inestable, sino lo suficientemente firme para asegurar la dirección.

\section{Motivador}

En términos generales, los objetivos fuera del alcance de las personas no son objetivos motivadores ni son logrados fácilmente. En la mayoría de las empresas, los objetivos motivadores son aquellos que son un poco agresivos, van un poco más allá de los límites de lo posible. Ciertas investigaciones han confirmado que objetivos específicos aumentan la productividad superior a aquella de objetivos fáciles de lograr.

\section{Comprensible}

Los objetivos deberían establecerse con palabras muy sencillas y comprensible. Sin embargo, no importando cómo están formulados, los direc- tores que los fijaron deberían asegurarse de que sean comprendidos por todos aquellos involucrados con sus logros. Este punto puede aparentar no tener fundamento, pero es uno muy importante; un estudio sobre peligros latentes en la planeación concluyó que los objetivos malentendidos fueron la principal causa de quejas entre los directivos.

\section{Obligación}

Una vez que se haya llegado a un acuerdo respecto a los objetivos, debería existir una obligación para hacer lo necesario y razonable y así lograrlos.

\section{Participación de las personas}

Los mejores resultados se logran cuando aquellos responsables del logro de objetivos pueden participar en el establecimiento de los mismos. Este punto se refiere más bien a empresas grandes, descentralizadas que a compañías pequeñas.

Las personas que participan en la determinación de objetivos que deben lograr se sentirán más motivadas para hacerlo que aquellas que tienen poca ingerencia en esta área. Además, el involucramiento en el proceso de establecimiento ayuda a satisfacer las necesidades de las personas ya que éstas tienen que usar sus capacidades para promover los intereses, tanto los propios como los detallados, íntimos y sustanciales de los ejecutivos y del personal acerca de sus propias operaciones son muy superiores en general a aquellos de los altos directivos. En tales casos, la colaboración entre la alta dirección y los gerentes de divisiones al fijar los objetivos representa una gran ventaja.

\section{Relación}

Existen varios aspectos en cuanto a la relación. Primero, los objetivos deben relacionarse con los propósitos básicos. Segundo, los objetivos de diferentes partes de la empresa deben examinarse para que sean consistentes con y satisfagan los objetivos de la alta dirección.

\section{AGENCIAS DE VIAJES Y TURISMO}

Toda agencia de viajes y turismo independientemente de su clasificación debe de someterse al Decreto Supremo N. ${ }^{\circ}$ 026-2004-Mincetur el cual es el reglamento de agencias de viajes y turismo, este establece los requisitos y procedimientos para autorizar la prestación del servicio de agencia de viajes y turismo, define el concepto de agencias de viajes y turismo, establece las condiciones que deberán cumplir para la prestación del servicio. 
Establece la clasificación como: minorista, mayorista y operador de turismo, y precisa la obligatoriedad de presentar una declaración jurada que de cuenta el cumplimiento de requisitos mínimos. Entre ellos se menciona:

En su Artículo $3^{\circ}$.- Definiciones. Expresa lo siguiente:

Para los efectos del presente reglamento se entiende por:

a) Agencia de viajes y turismo: Persona natural o jurídica que se dedica en forma exclusiva al ejercicio de actividades de coordinación, mediación, producción, promoción, consultoría, organización y venta de servicios turísticos, pudiendo utilizar medios propios o contratados para la prestación de los mismos.

b) Clase: Modalidad bajo la cual la agencia de viajes y turismo presta sus servicios, de acuerdo a la clasificación establecida en el artículo $2^{\circ}$ del presente reglamento.

c) Agencia de viajes y turismo minorista: Aquella que vende directamente al turista pasajes y/o servicios turísticos no organizados; comercializa el producto de los operadores de turismo y de las agencias de viajes y turismo mayoristas. La agencia de viajes y turismo minorista no puede ofrecer sus productos a otras agencias de viajes y turismo.

d) Agencia de viajes y turismo mayorista: Aquella que proyecta, elabora y organiza todo tipo de servicios turísticos y viajes para ser ofrecidos a otras agencias de viajes y turismo, no pudiendo ofrecer ni vender sus productos directamente al turista.

e) Operador de turismo: Aquél que proyecta, elabora, diseña, organiza y opera sus productos y servicios dentro del territorio nacional, para ser ofrecidos y vendidos a través de las agencias de viajes y turismo mayoristas y agencias de viajes y turismo minoristas, pudiendo también ofrecerlos y venderlos directamente al turista.

f) Personal calificado: Persona que cuenta con formación, capacitación y/o experiencia demostrada para atender, asesorar y orientar a los turistas que soliciten la prestación de servicios de la agencia de viajes y turismo.

g) Turismo emisor: El realizado por nacionales en el exterior. h) Turismo interno: El realizado dentro del territorio nacional por turistas domiciliados en el país.

i) Turismo receptor: El realizado dentro del territorio nacional por turistas domiciliados en el exterior.

j) Inspector: Funcionario o servidor público autorizado por el órgano regional competente, para desarrollar las acciones de verificación y supervisión previstas en el presente reglamento.

En su Artículo $6^{\circ}$.- Actividades de las agencias de viajes y turismo expresa:

Son actividades de las agencias de viajes y turismo, independientemente de la clase bajo la cual presten sus servicios, las siguientes:

a) La promoción del turismo en los ámbitos nacional e internacional;

b) La representación de empresas intermediarias $o$ agencias de viajes y turismo no domiciliadas en el país;

c) La representación de líneas aéreas;

d) La representación de otros prestadores de servicios turísticos;

e) El alquiler de vehículos con y sin conductor;

f) El fletamento de aviones, barcos, autobuses, trenes especiales y otros medios de transporte;

g) La organización y promoción de congresos, convenciones y otros eventos similares;

h) La contratación de guías de turismo;

i) Otras vinculadas a los viajes de turismo.

6.1 De las agencias de viajes y turismo mayoristas:

La proyección, elaboración, organización y comercialización de programas y demás servicios turísticos, a nivel nacional e internacional.

6.2 De los operadores de turismo:

a) La proyección, elaboración, producción, organización, operación, asesoría y comercialización de programas y demás servicios turísticos, a nivel nacional e internacional;

b) La intermediación en la reserva y venta de boletos y pasajes en cualquier medio de transporte;

c) La intermediación en la reserva y contratación de hospedaje; 
d) Recepción y traslado de turistas;

e) Brindar orientación e información al turista;

f) Cambio de divisas, venta y cambio de cheques de viajeros;

g) Transporte de equipaje a través de terceros utilizando cualquier medio de transporte;

h) Tramitar pólizas de seguros de viaje, de pérdidas o deterioro de equipajes y otras que cubran los riesgos del viaje;

i) Alquiler de útiles y equipos para la práctica de diversas modalidades de turismo;

j) Representación de los turistas en los procedimientos necesarios para concretar los viajes, cumpliendo con las disposiciones legales vigentes;

k) Recuperación de impuestos que el turista paga en el exterior.

6.3 De las agencias de viajes y turismo minoristas:

a) La elaboración, asesoría y comercialización de programas y demás servicios turísticos, a nivel nacional e internacional;

b) Las indicadas en el punto 6.2, con excepción de la señalada en su literal a).

En su Artículo 7o.- Ejercicio de las actividades de las agencias de viajes y turismo expresa:

Las actividades señaladas en el artículo $6^{\circ}$ del presente reglamento serán prestadas en el territorio nacional por las agencias de viajes y turismo, sin perjuicio de la contratación directa de tales servicios, por parte de los turistas, con las empresas de transporte, establecimientos de hospedaje y otros prestadores de servicios turísticos.

\section{MÉTODOS Y RESULTADOS}

Acorde al Proyecto inicial, se ha determinado que la cantidad de Agencias de Viajes y Turismo existentes en Lima al año 2009 acorde al MINCETUR son de 2,383 agencias de viajes y turismo a nivel de la región Lima.

Un estudio de la Pontificia Universidad Católica del Perú determina que las mayores agencias de viajes y turismo a nivel de la región son de 44 agencias de viajes y turismo representativas de las cuales se ha tomado como referencia 10 de las mejores agencias que operan turismo receptivo, egresivo e interno.
Estas son:

ABERCROMBIE \& KENT PERU S.A.C.

AC TOURS S.A.C.

AMERICAN REPS S.R.LTDA.

CARLSON WAGONLIT PERU S.A

CARRUSEL REPRESENTACIONES S.A.C

COLTUR PERUANA DE TURISMO S A

CÓNDOR TRAVEL S. A.

CONTINENTAL TRAVEL S.A.C.

COSTAMAR TRAVEL CRUISE \& TOURS S.A.C.

DASATOUR S.A.C.

Durante el tiempo que duró el estudio constantemente se hizo:

Visitas a las agencias de viajes y turismo seleccionadas a fin de determinar sus avances en la gestión del presente año

Coordinación con el personal directivo para determinar cambios de políticas de gestion

Entrevistas realizadas a la gerencia antes del estudio para determinar indicadores pre y post estudio

Se utilizaron como herramientas las matrices clásicas del modelo de la planeación estratégica, tales como:

EFE (Matriz de Evaluación de Factores Externos)

MPC (Matriz del Perfil Competitivo)

EFI (Matriz de Evaluación de Factores Internos)

FODA (Matriz de Amenazas, Oportunidades, Debilidades y Fortalezas)

BCG (Matriz del Boston Consulting Group)

IE (Matriz Interna Externa)

PEYEA Matriz de la Posición Estratégica y Evaluación de la Acción)

GE (Matriz de la Gran estrategia)

MCPE (Matriz Cuantitativa de la Planeación Estratégica)

Los resultados reflejan lo siguiente:

Las empresas entrevistadas han compartido información y evidenciado signos de aplicación de planeamiento estratégico.

Se ha aplicado la metodología en las 10 mejores agencias de viajes y turismo. 
El estudio para el levantamiento de datos de factores e indicadores demoró un aproximado de 40 días.

Se realizó el monitoreo de las 10 empresas en forma constante hasta el mes de octubre.

En noviembre se solicitaron datos de los resultados obtenidos a las 10 agencias de viajes y turismo.

$30 \%$ de ellas, mantuvieron su misma tasa de crecimiento histórica, porque no manejan herramientas de Planeamiento Estratégico.

$70 \%$ de las agencias de viajes y turismo, manejan planeamiento estratégico, y que por esta razón llegaron a incrementar su productividad en el índice esperado de $17.50 \%$ acorde a los índices y resultados mostrados para este estudio.

\section{ANÁLISIS Y DISCUSIÓN}

Acorde a las metas del estudio, se ha estimado un incremento del 15\% en la comercialización de los servicios turísticos por parte de las agencias de viajes y turismo que implementan la Planeación Estratégica en su gestión, y los resultados arrojan un resultado mayor en 2.5 puntos.

El número de agencias de viajes y turismo, es pequeña, dado que la mayoría de ellas se encuentran en diferentes provincias o en los conos. Dado que no se ha utilizado encuestas, sino se determino entrevistar directamente a los directivos, en algunos casos las agencias de viajes y turismo, no querían dar información cuantitativa de esa naturaleza, por lo que se trabajo con datos porcentuales.

Acorde a los objetivos del estudio, se ha determinado que estas agencias de viaje y turismo, mejoran sus indices de productividad traducido en utilidades y aumento de clientes gracias al uso de herramientas de gestion empresarial de planeación estratégica

\section{CONCLUSIONES}

a) Se demuestra nuestra hipótesis que la planeación estratégica mejora la productividad en las agencias de viaje y turismo.

b) Podría ampliarse este estudio no solo a agencias de viaje y turismo en Lima, sino poder dividirlo por segmentos para poder compararlos con agencias de viaje y turismo tipo Mypes c) El estudio también puede abarcar no solo Agencias de Viaje y Turismo, sino Establecimientos de hospedaje, restaurantes, y cualquier otro prestatario de servicios turísticos.

d) La prosperidad de agencias de viaje y turismo se ven reflejados en la llegada de pasajeros nacionales e internacionales acorde a los datos emitidos por el Ministerio de Interior (Migraciones) y el Mincetur.

e) Se propondrá estos resultados para ampliarlos en otro estudio para llevarlo a cabo a nivel nacional

f) De este estudio se escribirá un artículo de investigación que permitirá a los alumnos de pre grado de las EAP de Administración en Turismo la realización de mayores tesis de pre grado y al mismo tiempo de post grado.

g) Sugerir al decanato de la Facultad de Ciencias Administrativas, la creación de una agencia de viaje y turismo - escuela para que nuestros alumnos puedan desarrollar sus prácticas pre profesionales

\section{BIBLIOGRAFÍA}

ACKOFF, Russell L. (1995): "Planificación De La Empresa Del Futuro". México. Editorial Limusa S.A. De C.V.

BATEMAN, Thomas S. (2005): "Administración: Un Nuevo Panorama Competitivo” México, D.F. Mcgraw-Hill.

BATEMAN, Thomas S. (2009): “Administración: Liderazgo Y Colaboración En Un Mundo Competitivo". México, D.F.: Mcgraw-Hill.

BERNAL TORRES, César A. (2007): “Introducción a la administración de las organizaciones: enfoque global e Integra". México, D.F.: Pearson.

BONIFACE, Priscilia. (1995): "Managing Quality Cultural Tourism”. Routledge London/New York.

BURNS, Peter; HOLDEN, Andrew. (1995): “Tourism: A New Perspective”. Prentice hall. London.

CERTO, Samuel / PETER, J.Paul / OTTENSMEYER, Edward. (1996): "Dirección Y Administración Estratégicas". 3era. Ed. Rev. Corregida Y Aumentada Madrid / Mosby-Doyma Libros, S.A. -División Irwin.

CERTO, Samuel C. (2001): "Administración Moder- 
na: Diversidad, Calidad, Ética, \& El Entorno Global”. Bogotá. Prentice Hall.

CHIAVENATO, Adalberto (2007): "Introducción A La Teoría General De La Administración”. México, D.F.: Mcgraw-Hil.

DAVID, Fred D. (2008): "Conceptos De Administración Estratégica”. México, D.F.: Pearson, 11 edicion.

DRUKER, Peter (2006). "Druker Para Todos Los Días: 366 Días De Reflexiones Clave Para Acertar En Sus Negocios”. Bogota: Editorial Norma .

DRUKER, Peter. (2003): "La Gerencia En La Sociedad Futura”. Colombia. Editorial Norma S.A.

GARTNER, William C. (1996): “Tourism Development, Principles, Processes And Policies". New York : Van Nostrand Reinhold.

GEE, Chuck Y. (1997): “The Travel Industry”. 3a. ed. Van Nostrand Reinhold. New York.

HARRISON, Lynn C. (1996): "Practicing Responsible Tourism. International Case Studies In Tourism Planning, Policy And Development". Wiley. New York.

HERNÁNDEZ y RODRÍGUEZ, Sergio (2008): "Administración: Teoría, Proceso, Áreas Funcionales Y Estrategias Para La Competitividad". México, D. F.: Mcgraw-Hill.

HILL, Charles W.L. / JONES Gareth R. (1996): "Administración Estratégica, Un Enfoque Integrado". 3era. Edición Ampliada. Mc Graw-Hill Interamericana, S.A.

HITT, Michael A. (2008): "Administración Estratégica: Competitividad Y Globalización. Conceptos Y Caso". México, D.F.: Thomson.
HITT, Michael A., Black, J. Stewart Y Porter, Lyman W. (2006): "Administración". México, D. F.: Pearson.

JONES, Gareth R. (2006): "Administración Contemporánea”. México, D.F.: Mcgraw-Hil.

KOONTZ, Harold (2008): “Administración: Una Perspectiva Global Y Empresarial”. México, D.F.: Mcgraw-Hill.

MAYURÍ B. Jorge V. (2010): "Separata Del Curso: Planeamiento Estratégico"

MINTZBERG, Henry (2005): "Directivos No Mas: Una Visión Crítica De Empresas Y Formación”. Barcelona: Deusto.

PORTER, Michael E. (2007): "Estrategia Competitiva: Técnicas Para El Análisis De Los Sectores Industriales Y De La Competencia”. México, D. F.: Grupo Editorial Patria.

RÍOS SZALAY, Adalberto. (2007): "Orígenes De La Administración: Tendencias De Desarrollo En El Siglo XXI". México, D.F.: Trillas, 2007.

ROBBINS, Stephen P. (2002): "Fundamentos De Administración: Conceptos Esenciales Y Aplicaciones”. México, D.F.: Pearson.

ROBBINS, Stephen P. (2005): "Administración". México: Pearson.

ROBBINS, Stephen P. (2009): "Fundamentos De Administración: Conceptos Esenciales Y Aplicaciones”. México, D.F.: Pearson.

STONER, James / FREEMAN R. Edward / GILBERT Daniel R. Jr. (1996): "Administración”. México, Prentice Hall, 6ta. Ed.

THOMPSON, Arthur A. (2007): "Administración Estratégica: Teoría Y Casos”. México, D.F.: Mcgraw-Hill. 
ANEXOS

Fuente: MININTER - DIGEMIN

\section{PERÚ: LLEGADAS INTERNACIONALES, 2008}

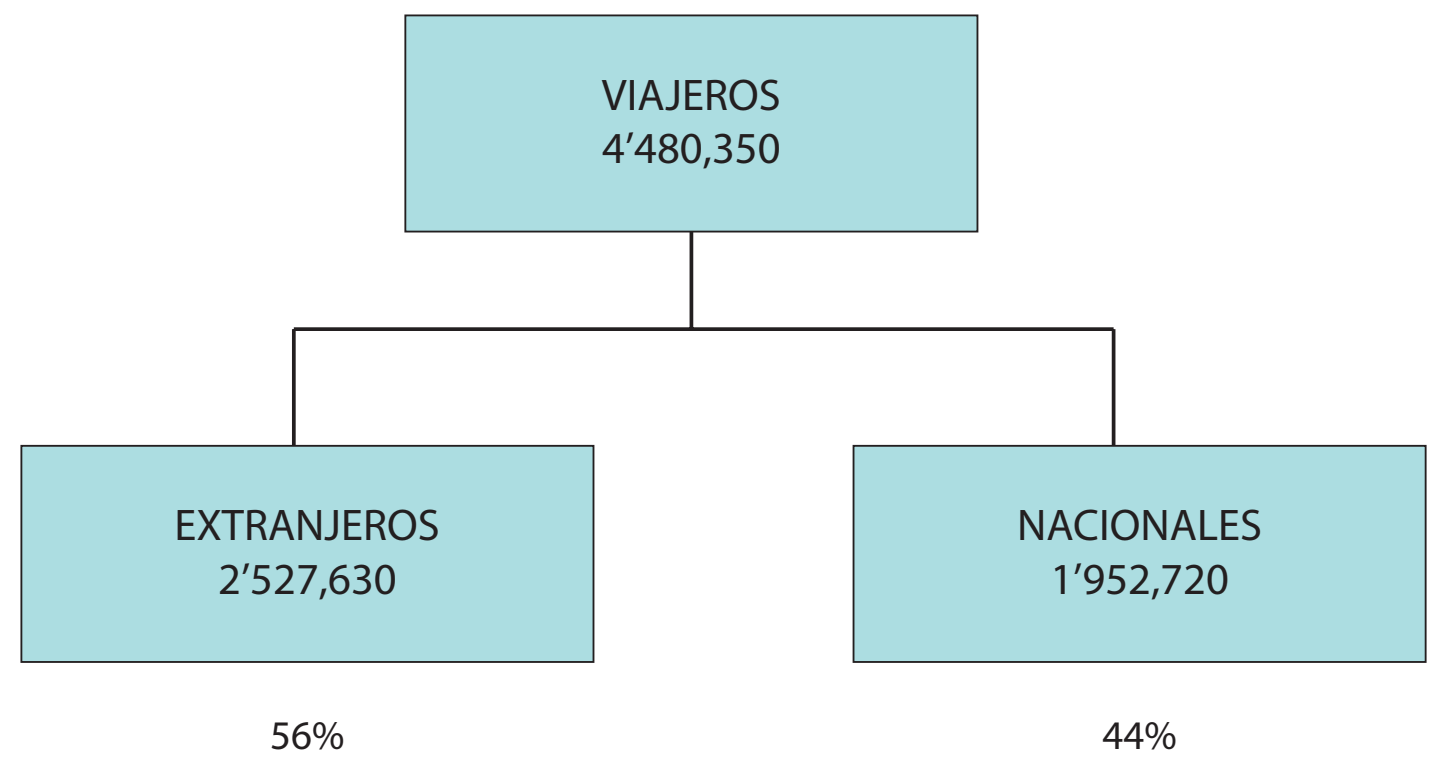

\section{PERÚ: LLEGADA DE EXTRANJEROS, 2008}

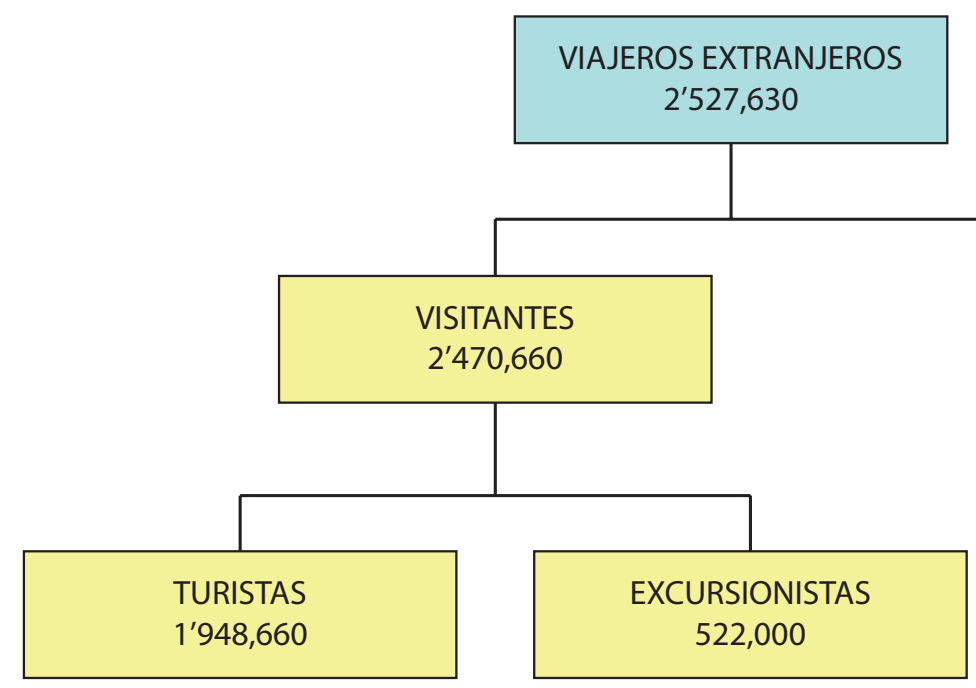




\section{TOTAL DE TURISTAS AL PERÚ, 2008}

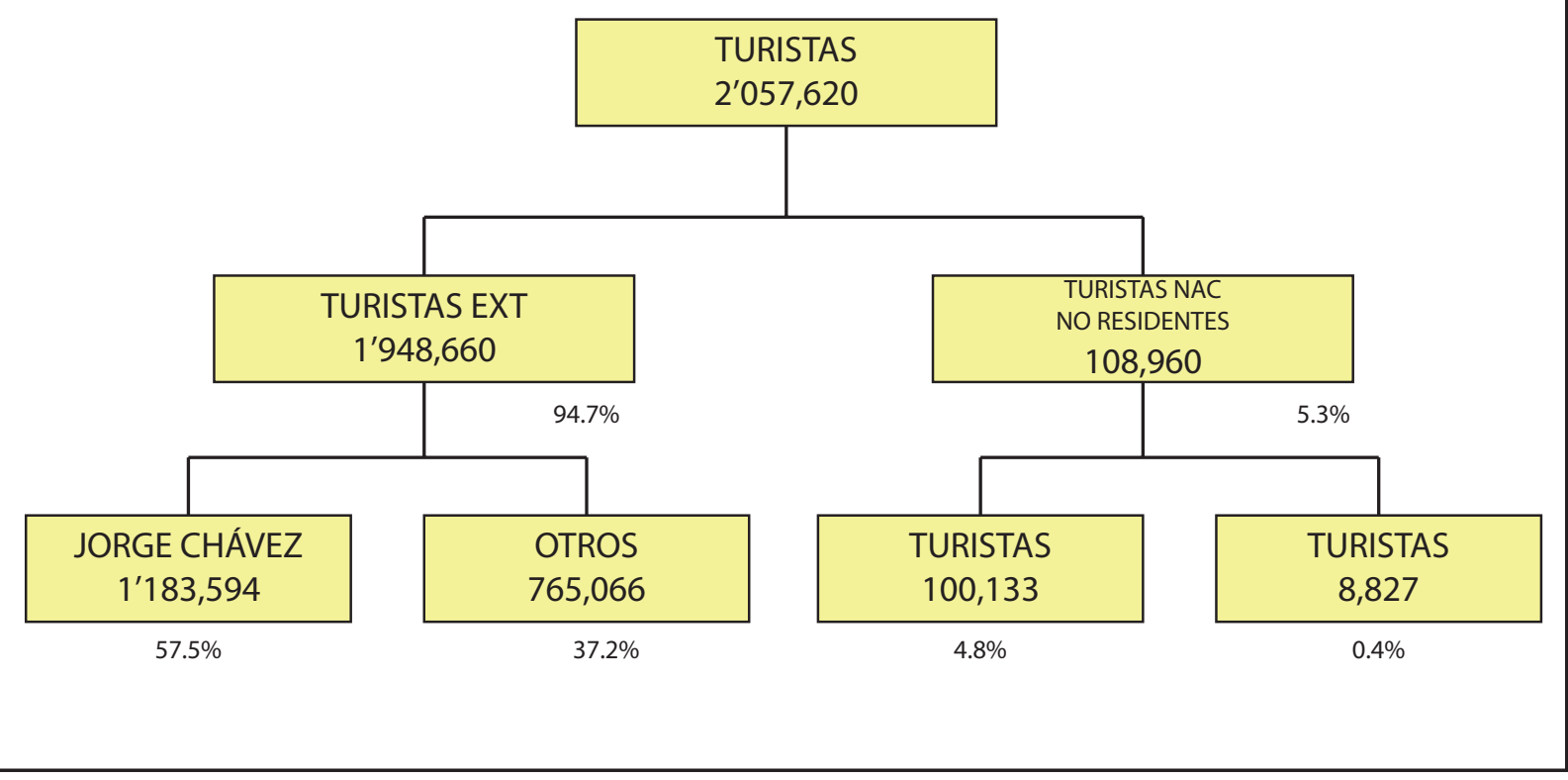

\section{¿Por qué disminuyó la llegada de visitantes internacionales al Perú en el $4^{\circ}$ trimestre 2008?... ¿Debido a la crisis mundial?}

\begin{tabular}{|l|l|}
\hline \multicolumn{1}{|c|}{ FECHA DEL ACONTECIMIENTO } & \multicolumn{1}{c|}{ CAUSAS } \\
\hline Del 1 al 2 de octubre 2008 & $\begin{array}{l}\text { Moquegua: Paro de 48 horas por la justa distribución del Canon } \\
\text { Minero. } \\
\text { Del } 1 \text { al } 3 \text { de octubre } 2008\end{array}$ \\
29 de octubre 2008 & $\begin{array}{l}\text { Tacna: Paro de } 48 \text { horas por el cumplimiento a la actual distribución } \\
\text { del Canon Minero. } \\
\text { Moquegua: Paro indefinido por la aprobación del Proyecto de Ley } \\
\text { 2713. Modificación en el sistema de distribución del Canon Minero. } \\
\text { Moquegua: Manifestación por el levantamiento de la huelga indefi- } \\
\text { nida. Se modificó el sistema de distribución del Canon Minero. } \\
\text { Tacna: Paro por el rechazo al proyecto de Ley que modifica la distri- } \\
\text { bución del Canon Minero. } \\
\text { Tacna: Paro por la distribución del Canon Minero. }\end{array}$ \\
$\begin{array}{l}\text { Del } 20 \text { de octubre } 2008 \text { al } 12 \text { de no- } \\
\text { viembre } 2008\end{array}$ & \\
\hline
\end{tabular}




\section{PERÚ: TURISTAS EXTRANJEROS 2004-2008}

\begin{tabular}{|c|c|c|c|c|c|c|}
\hline & 2004 & $2005 \mathrm{p} /$ & $2006 \mathrm{p} /$ & $2007 \mathrm{p} /$ & $2008 \mathrm{p} /$ & \\
\hline CHILE & 301,024 & 338,929 & 415,106 & 464,678 & 445,590 & $-4.1 \%$ \\
\hline EEUU & 243,790 & 293,240 & 297,317 & 321,317 & 370,945 & $15.3 \%$ \\
\hline ECUADOR & 110,294 & 100,808 & 111,239 & 118,625 & 126,852 & $6.9 \%$ \\
\hline ARGENTINA & 46,035 & 56,232 & 63,543 & 77,712 & 90,914 & $17.0 \%$ \\
\hline BOLIVIA & 65,906 & 71,718 & 84,068 & 86,335 & 85,245 & $-1.3 \%$ \\
\hline COLOMBIA & 39,026 & 48,365 & 53,639 & 62,335 & 75,538 & $221.6 \%$ \\
\hline ESPAÑA & 43,023 & 58,785 & 62,289 & 63,371 & 74,196 & $17.1 \%$ \\
\hline REINO UNIDO & 49,396 & 58,712 & 59,876 & 66,488 & 65,800 & $-1.0 \%$ \\
\hline BRASIL & 33,327 & 43,291 & 44,092 & 52,515 & 62,902 & $19.8 \%$ \\
\hline FRANCIA & 43,848 & 52,050 & 53,518 & 58,713 & 62,901 & $7.1 \%$ \\
\hline ALEMANIA & 34,846 & 42,565 & 42,663 & 49,312 & 50,737 & $2.9 \%$ \\
\hline CANADÁ & 25,610 & 33,933 & 40,007 & 40,983 & 50,306 & $22.7 \%$ \\
\hline JAPÓN & 27,326 & 32,553 & 33,925 & 38,424 & 42,745 & $11.2 \%$ \\
\hline ITALIA & 24,296 & 27,258 & 26,755 & 31,013 & 31,410 & $1.3 \%$ \\
\hline VENEZUELA & 16,357 & 20,995 & 20,868 & 24,112 & 28,937 & $20.0 \%$ \\
\hline AUSTRALIA & 16,424 & 20,225 & 22,081 & 25,161 & 28,397 & $12.9 \%$ \\
\hline MÉXICO & 23,510 & 27,951 & 28,168 & 30,259 & 28,197 & $-6.8 \%$ \\
\hline HOLANDA & 16,290 & 17,346 & 17,681 & 23,908 & 26,803 & $12.1 \%$ \\
\hline RESTO & 116,311 & 141,546 & 157,910 & 177,041 & 200,245 & $13.1 \%$ \\
\hline & $1,276,639$ & $1,486,502$ & $1,634,745$ & $1,812,384$ & $1,948,660$ & \\
\hline
\end{tabular}

